\title{
Cervical Ligamentous Injury with Associated Hydrocephalus Secondary to Posterior Fossa Subdural Hemorrhage: A Case Report
}

\author{
Ahmed A. Cheema ${ }^{1}$ Timothy A. Puckett ${ }^{2}$ Michael D. Martin ${ }^{1}$ \\ ${ }^{1}$ Department of Neurosurgery, University of Oklahoma-Health Science \\ Centers, Oklahoma City, Oklahoma, United States \\ 2 Department of Orthopedics, University of Oklahoma-Health Science \\ Centers, Oklahoma City, Oklahoma, United States \\ Address for correspondence Ahmed A. Cheema, MD, Department of \\ Neurosurgery, University of Oklahoma-Health Science Centers, 1000 \\ N. Lincoln Boulevard, Suite 400, Oklahoma City, OK 73104, \\ United States (e-mail: Michael-Martin@ouhsc.edu).
}

J Neurol Surg Rep 2014;75:e89-e92.

\begin{abstract}
Keywords

- subdural hematoma

- hydrocephalus

- cervical injury

Traumatic subdural hematomas (SDHs) in the posterior fossa in conjunction with cervical spinal hematomas are rare entities. In this case, we present a 22-year old man who presented to our institution with ligamentous injury at the cervicomedullary junction, associated with posterior fossa SDH not discovered initially, who on serial computed tomography scans was found to have symptomatic obstructive hydrocephalus and was successfully treated with SDH evacuation and stabilization of the cervicomedullary junction with instrumentation.
\end{abstract}

\section{Introduction}

Traumatic posterior fossa subdural hematomas (SDHs) in the adult population are a rarity, with a reported incidence $<1 \%{ }^{1}$ Its incidence with concurrent cervical spine injury is still unknown. Cases have been reported with lumbago and headaches secondary to chronic SDH collections in the lumbar spine associated with secondary findings of intracranial SDHs. ${ }^{2-5}$ These case reports stress the need for a close evaluation of patients presenting with mechanisms that can also cause intracranial SDH with complaints of headaches and lethargy in addition to evaluation of the spine. We report a case of a 22-year-old man involved in a motor vehicle collision who had a reported negative computed tomography (CT) scan for any hematoma on the initial traumatic evaluation. However, he was later found to have an SDH on the evaluation of new-onset lethargy and headaches.

\section{Case Presentation}

The patient is a 22-year-old man who was evaluated at an outside hospital after a motor vehicle collision while intoxicated and was found to have a ligamentous disruption in the upper cervical spine region. He continued to be followed closely at the outside hospital with increasing complaints of headaches and sleepiness and was transferred to our institution with the discovery of increasing ventricular prominence on serial imaging with concerns for hydrocephalus. Throughout the hospitalization at the outside institution he was neurologically intact. The patient otherwise is healthy and does not take any medications.

On presentation to our institution, the patient continued with complaints of headaches; however, he had symmetrical $5 / 5$ strength bilaterally with no bulbar symptoms. Considering no changes in the characteristics of headaches associated with increasing lethargy, a head CT and cervical spine magnetic resonance imaging were obtained that showed worsening of the ventriculomegaly with transependymal flow associated with SDH not seen on the initial imaging modalities (-Figs. 1 and 2, which was concerning for obstructive hydrocephalus. In addition, the serial CT scans showed cisternal effacement ( - Fig. 3), for which the patient was taken to the operating room for evacuation of the SDH, along with stabilization of the cervical spine with instrumentation.

Intraoperatively, once the dura was opened, a chronicappearing hematoma was encountered that was evacuated
License terms

Stuttgart · New York

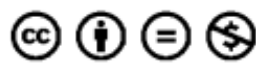

received

October 27, 2013

accepted

January 24, 2014

published online

June 4, 2014
DOI http://dx.doi.org/

10.1055/s-0034-1372473. ISSN 2193-6358. 


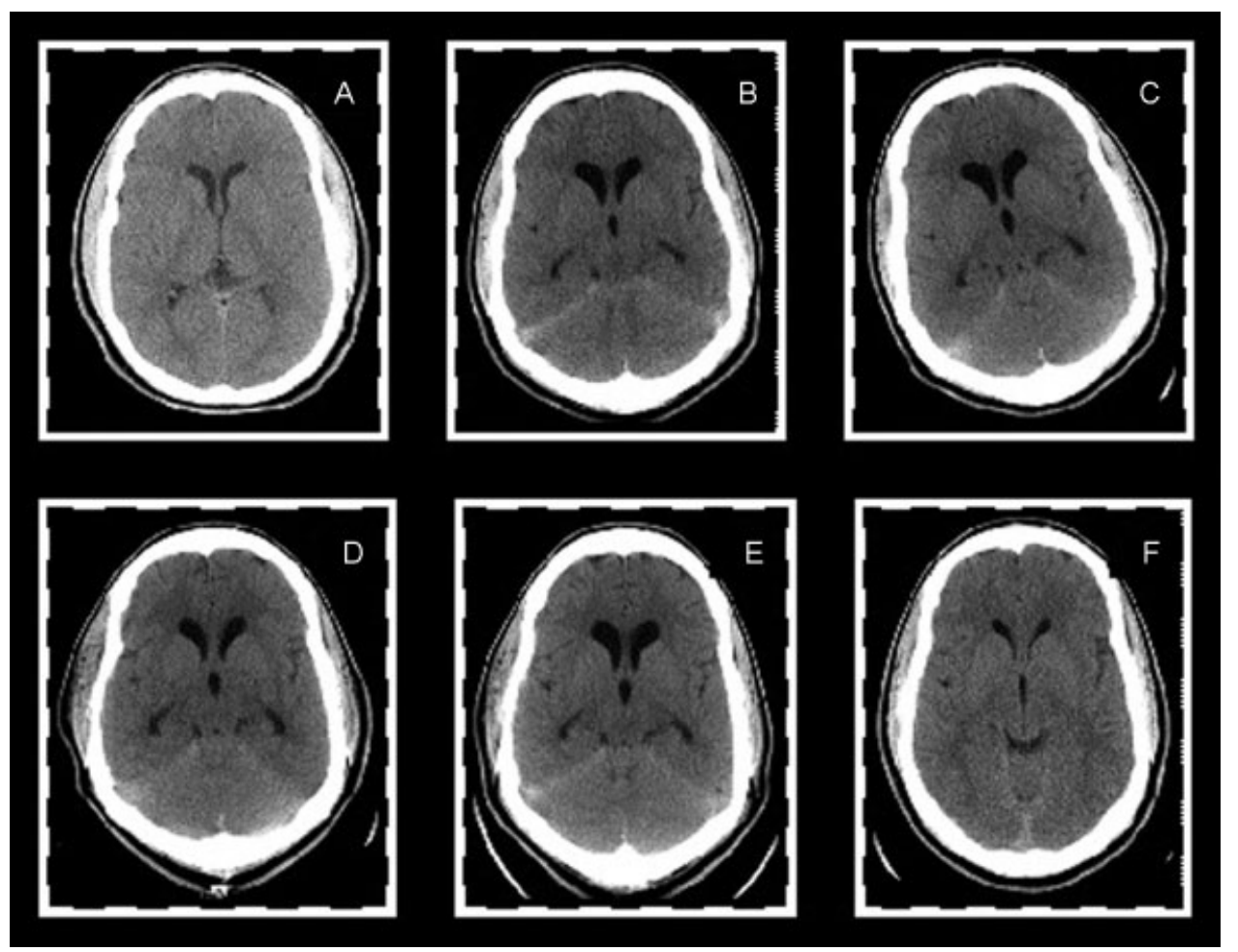

Fig. 1 Serial head computed tomography scans for the patient showing worsening and then improvement of the hydrocephalus postoperatively. (A) On presentation. (B) Subdural hematoma (SDH) with ventriculomegaly. (C) SDH with worsening ventriculomegaly done preoperatively. (D) Postoperative day 1. (E) Postoperative day 4. (F) Three months postoperative.
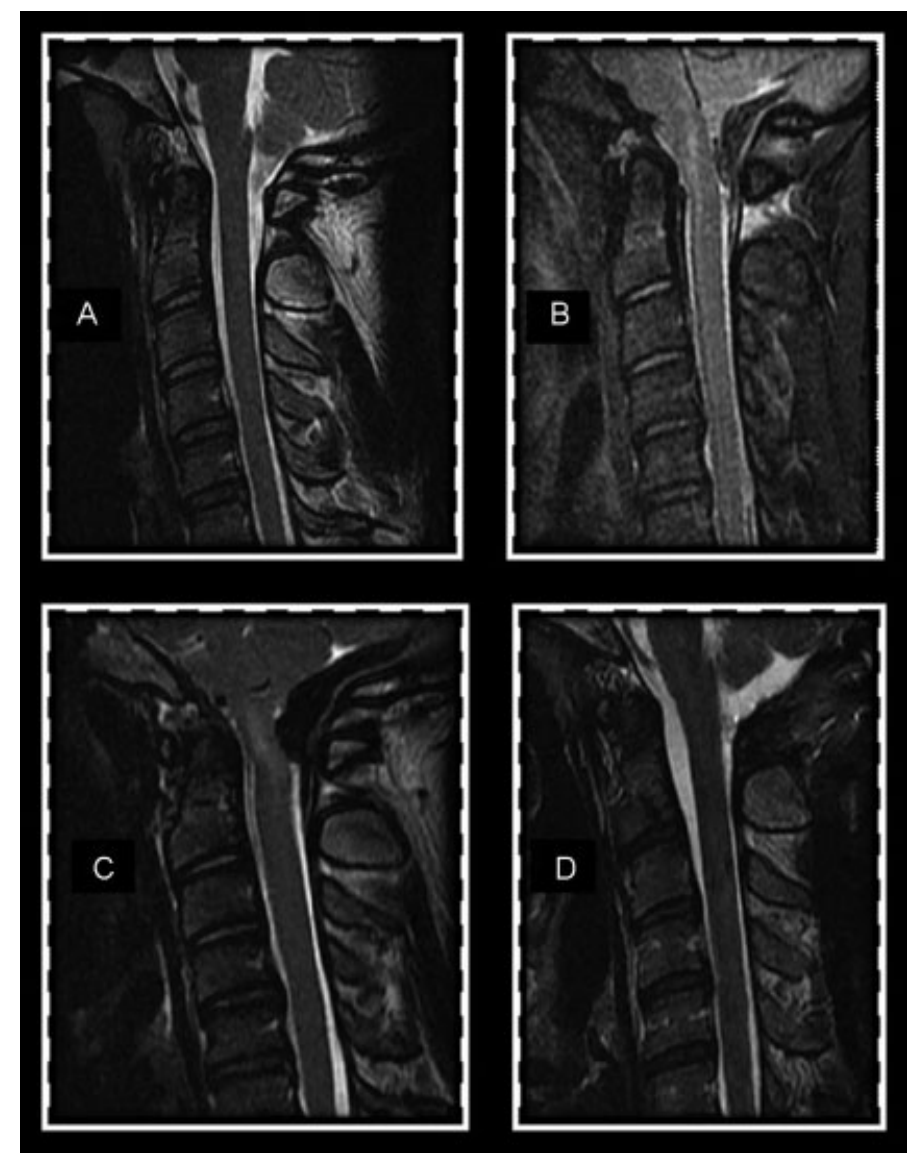

Fig. 2 Cervical spine magnetic resonance imaging showing ligamentous injury with subdural hematoma (SDH) and resolution of SDH postoperatively. (A) On presentation, no signs of SDH. (B) On presentation of lethargy showing SDH and cord signal changes. (C) On presentation of lethargy showing SDH and cord signal changes. (D) Three-months postoperative showing resolution of SDH. 


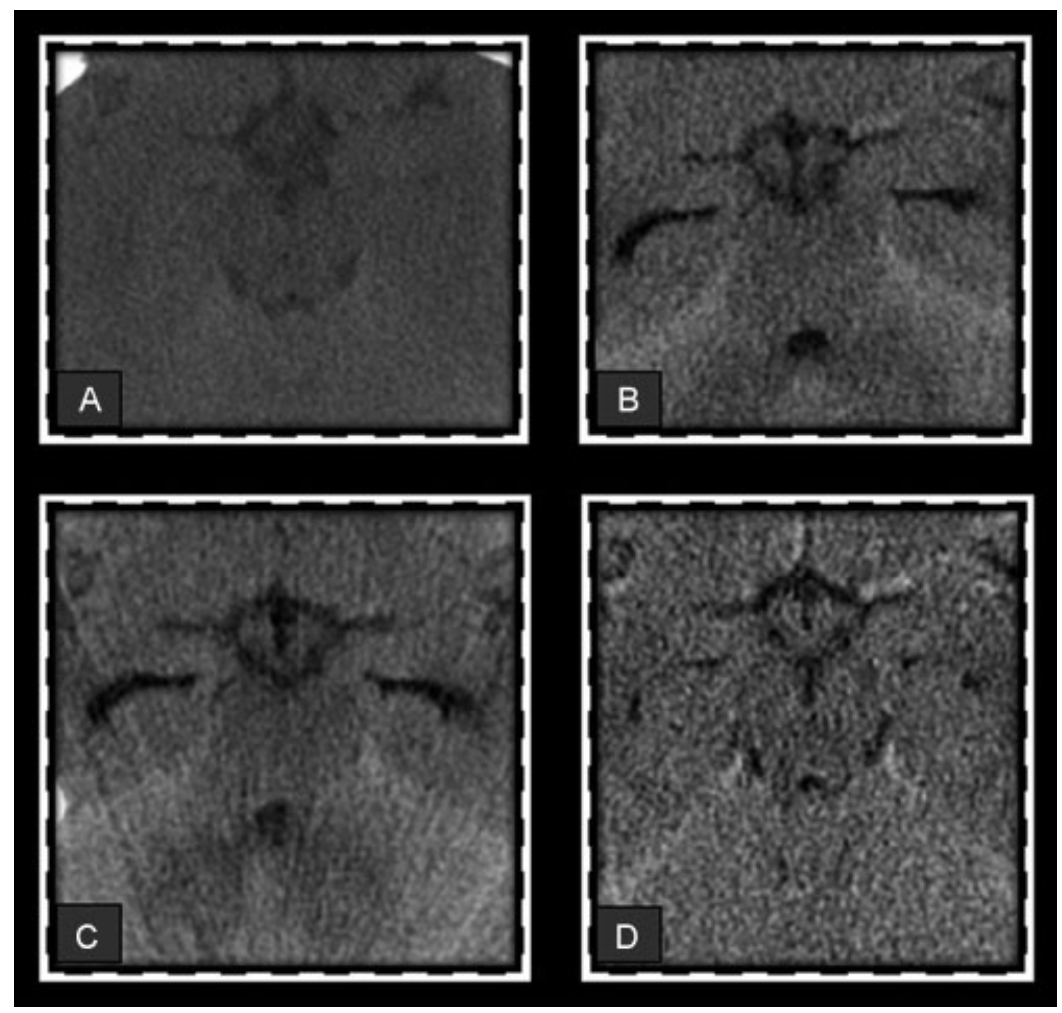

Fig. 3 Computed tomography scans showing cisternal compression with temporal horn enlargement that is improved postoperatively. (A) On presentation, no temporal horn enlargement. (B) On presentation of lethargy showing cisternal compression and temporal horn enlargement. (C) Postoperative day 4 showing temporal horn enlargement with cisternal effacement. (D) Three months postoperative showing improvement of temporal horn enlargement and cisternal effacement.

without difficulties. The dura was primarily closed, and the patient was followed clinically with serial CT scans on postoperative days 1 and 4, and at 3 months, which showed a decrease in the cisternal effacement and also clinical improvement in the patient's headaches and lethargy (-Figs. 1 and $\mathbf{3}$ ).

\section{Discussion}

Posterior fossa SDH represents a rare entity with diagnostic and treatment challenges. In the reported literature, $1 \%$ of SDHs are located in the posterior fossa. ${ }^{6}$ Direct trauma to the occipital region has been repeatedly associated with hemorrhage in the posterior fossa whether it is subarachnoid, subdural, or epidural in its characteristics. ${ }^{7}$ Mass lesions in the posterior fossa have a $23 \%$ incidence of acute hydrocephalus. There have been reports of both conservative and surgical management of these hematomas. However, most neurosurgeons, in their small patient population reports, have proposed early treatment of these lesions with hematoma evacuation and decompression.

Various associated findings have been reported in patients with posterior fossa hemorrhagic lesions. These include diplopia, headaches, lethargy, nuchal rigidity, facial nerve palsies, and locked-in syndrome. ${ }^{8-11}$ With mortality rates of 50 to $71 \%$ reported, these symptoms warrant careful evaluation and early imaging to rule out any intracranial lesions. ${ }^{12}$ Particularly in patients with cervical spinal liga- mentous disruption acting as a distracting lesion, signs and symptoms concerning for intracranial pathology should be followed closely.

Borzone et al reported that $60 \%$ of patients with a known $\mathrm{SDH}$ in the posterior fossa clinically deteriorated within the first 24 hours. ${ }^{13,14}$ It presents a clinical dilemma in our case because an initial head CT did not show any intracranial injury; however, considering ligamentous injury and mechanism of injury, the delayed presentation of intracranial hemorrhage was entertained based on clinical presentation. The outcomes of a patient in a similar situation was stratified in a case series based on the Glasgow Coma Scale (GCS). ${ }^{15,16}$ In patients with a GCS score $>8$, outcome was favorable in $75 \%$ of cases. On the contrary, of the patients with a GCS score $<8,91.6 \%$ had poor outcomes. Based on these limited studies, it is strongly recommended that patient with a posterior fossa hematoma be considered for surgical evacuation for the best functional outcome even in an agonal condition.

\section{Conclusion}

Patients with a history of trauma with declining neurologic status warrant close monitoring and imaging to evaluate for intracranial lesions. In our case, the patient presented with cervical spine injury but no intracranial injuries on initial head CT. However, the patient developed hydrocephalus that was successfully treated with posterior fossa decompression 
and hematoma evacuation. Care should be taken in the evaluation of patients with cranovertebral junction injuries with declining arousal because it may indicate an intracranial pathology. Posterior fossa symptomatic subdural hemorrhages are rare entities. ${ }^{17}$ If they are identified and treated early, they have promising results with good functional outcomes as we experienced in our particular case.

\section{References}

1 Takeuchi S, Takasato Y, Wada K, et al. Traumatic posterior fossa subdural hematomas. J Trauma Acute Care Surg 2012;72(2): 480-486

2 Nagashima H, Tanida A, Hayashi I, et al. Spinal subdural haematoma concurrent with cranial subdural haematoma: report of two cases and review of literature. Br J Neurosurg 2010;24(5):537-541

3 Nakajima M, Fukuda S, Ikeda H, Suzuki Y. Chronic spinal subdural hematoma associated with chronic intracranial subdural hematoma: a case report. [in Japanese]. No Shinkei Geka 2009;37(10):1013-1017

4 Hagihara N, Abe T, Kojima K, Watanabe M, Tabuchi K. Coexistence of cranial and spinal subdural hematomas: case report. Neurol Med Chir (Tokyo) 2010;50(4):333-335

5 Yamaguchi S, Kurisu K, Arita K, Takeda M, Tani I, Araki O. Simultaneous cranial and spinal subdural hematoma. Neurol Med Chir (Tokyo) 2005;45(12):645-649

6 Isla A, Alvarez F, Manrique M, Castro A, Amaya C, Blázquez MG. Posterior fossa subdural hematoma. J Neurosurg Sci 1987;31(2): 67-69

7 Karasawa H, Furuya H, Naito H, Sugiyama K, Ueno J, Kin H. Acute hydrocephalus in posterior fossa injury. J Neurosurg 1997;86(4): 629-632
8 Catalá-Antúnez I, de Quintana-Schmidt C, Clavel-Laria P, MontesGraciano G, Molet-Teixidó J. Abducens nerve palsy due to posttraumatic retroclival and spinal subdural hematoma: case report and literature review. [in Spanish]. Neurocirugia (Astur) 2011; 22(4):337-341

9 Sedney CL, Coger BR, Bailes JE. Posterior fossa subdural hematoma resulting in locked-in syndrome: case report. Neurosurgery 2011; 69(2):E497-E500

10 Gulsen S, Sonmez E, Yilmaz C, Altinors N. Traumatic acute subdural hematoma extending from the posterior cranial fossa to the cerebellopontine angle. J Korean Neurosurg Soc 2009;46(3): 277-280

11 Milo R, Razon N, Schiffer J. Delayed epidural hematoma. A review. Acta Neurochir (Wien) 1987;84(1-2):13-23

12 Raftopoulos C, Reuse CH, Chaskis C, Brotchi J. Acute subdural hematoma of the posterior fossa. A case report and a review of the relevant literature. Clin Neurol Neurosurg 1990;92(1):57-62

13 Borzone M, Rivano C, Altomonte M, Baldini M. Acute traumatic posterior fossa subdural haematomas. Acta Neurochir (Wien) 1995;135(1-2):32-37

14 Sridhar K, Venkateswara PG, Ramakrishnaiah S, Iyer V. Posttraumatic retroclival acute subdural hematoma: report of two cases and review of literature. Neurol India 2010;58(6):945-948

15 d'Avella D, Servadei F, Scerrati M, et al. Traumatic acute subdural haematomas of the posterior fossa: clinicoradiological analysis of 24 patients. Acta Neurochir (Wien) 2003;145(12):1037-1044; discussion 1044

16 Hećimović I, Blagus G, Kristek B, Rukovanjski M, Vranković D. Successful treatment of traumatic acute posterior fossa subdural hematoma: report of two cases. Surg Neurol 1999;51(3):247-251

17 Shiraga S, Akai T, Iida T, lizuka H. Subdural hematoma at the cranio-vertebral junction following head trauma: a case report. [in Japanese]. No Shinkei Geka 2010;38(4):365-370 